\title{
ON EXTENDED TYPE I GENERALIZED LOGISTIC DISTRIBUTION
}

\author{
A. K. OLAPADE
}

Received 2 September 2003

We consider a form of generalized logistic distribution which is called extended type I generalized logistic distribution. Some theorems that relate the distribution to some other statistical distributions are established. A possible application of one of the theorems is included.

2000 Mathematics Subject Classification: 62E15, 62E10.

1. Introduction. The probability density function of a random variable that has logistic distribution is

$$
f_{X}(x)=\frac{e^{-x}}{\left(1+e^{-x}\right)^{2}}, \quad-\infty<x<\infty
$$

and the corresponding cumulative distribution function is given by

$$
F_{X}(x)=\left(1+e^{-x}\right)^{-1}, \quad-\infty<x<\infty .
$$

The importance of the logistic distribution has already been felt in many areas of human endeavour. Verhulst [12] used it in economic and demographic studies. Berkson $[3,4,5]$ used the distribution extensively in analyzing bioassay and quantal response data. The works $[6,9,10,11,8]$ are a few of many publications on logistic distribution.

The simplicity of the logistic distribution and its importance as a growth curve have made it one of the many important statistical distributions. The shape of the logistic distribution that is similar to that of the normal distribution makes it simpler and also profitable on suitable occasions to replace the normal distribution by the logistic distribution with negligible errors in the respective theories.

Balakrishnan and Leung [2] show the probability density function of a random variable $X$ that has type I generalized logistic distribution. It is given by

$$
f_{X}(x ; b)=\frac{b e^{-x}}{\left(1+e^{-x}\right)^{b+1}}, \quad-\infty<x<\infty, b>0 .
$$

The corresponding cumulative distribution function is

$$
F_{X}(x ; b)=\left(1+e^{-x}\right)^{-b}, \quad-\infty<x<\infty, b>0,
$$

and the characteristic function of $X$ is

$$
\phi_{X}(t)=\frac{\Gamma(1-i t) \Gamma(b+i t)}{\Gamma(b)} .
$$


The means, variances, and covariances of order statistics from the type I generalized logistic distribution have been tabulated for some values of $b$ in [1]. Wu et al. [13] proposed an extended form of the generalized logistic distribution which is referred to as the five-parameter generalized logistic distribution. Its density function is given by

$$
\begin{aligned}
& f_{X}(x ; \mu, \sigma, \lambda, \phi, m) \\
& \quad=\frac{\lambda^{\phi}}{\sigma B(\phi, m)}\left[\exp \left(\frac{x-\mu}{\sigma}\right)\right]^{m}\left[\lambda+\exp \left(\frac{x-\mu}{\sigma}\right)\right]^{-(\phi+m)}, \quad-\infty<x<\infty,
\end{aligned}
$$

where $-\infty<\mu<\infty, \lambda>0, \phi>0, \sigma>0, m>0$. Several properties of this distribution such as moments are examined and some applications are discussed in [13].

In this paper, we consider a form of generalized logistic distribution density function that generalizes the type I generalized logistic distribution of Balakrishnan and Leung [2]. The new function, which is a particular case of the general case considered in [13], is called extended type I generalized logistic distribution.

2. Extended type I generalized logistic distribution. As mentioned above, Wu et al. [13] presented a generalized logistic distribution with density function (1.6). Putting $\mu=0$ and $\sigma=1$ and working with $-X$ instead of $X$, its density function can be written as

$$
f_{X}(x ; \lambda, \phi, m)=\frac{\lambda^{\phi}}{B(\phi, m)} \frac{e^{-m x}}{\left(\lambda+e^{-x}\right)^{\phi+m}}, \quad-\infty<x<\infty, \lambda>0, \phi>0, m>0 .
$$

In this section, we will derive a form of generalized logistic distribution which is a special case of the one in (2.1) as (1.3) is a special case of the generalized logistic distribution in [6].

Let $X$ be a continuously distributed random variable with one-parameter Gumbel density function

$$
f_{X}(x ; \alpha)=\alpha e^{-x} \exp \left(-\alpha e^{-x}\right), \quad-\infty<x<\infty, \alpha>0 .
$$

Assuming that $\alpha$ has a gamma distribution with probability density function

$$
h(\alpha ; \lambda, p)=\frac{\lambda^{p}}{\Gamma(p)} \alpha^{p-1} \exp (-\lambda \alpha), \quad p>0, \lambda>0 .
$$

We obtain the probability density function of the compound distribution using (2.2) and (2.3) as

$$
f_{X}(x ; \lambda, p)=\int_{0}^{\infty} f_{X}(x ; \alpha) h(\alpha ; \lambda, p) d \alpha=\frac{p \lambda^{p} e^{-x}}{\left(\lambda+e^{-x}\right)^{p+1}} .
$$

The function in (2.4) is what we refer to as the extended type I generalized logistic distribution density function. Equation (2.4) corresponds to $m=1, \phi=p$ in (2.1).

The corresponding cumulative distribution function is

$$
F_{X}(x ; \lambda, p)=\frac{\lambda^{p}}{\left(\lambda+e^{-x}\right)^{p}} .
$$


When $p=\lambda=1$, we have the ordinary logistic distribution and when $\lambda=1$, we have the type I generalized logistic distribution of Balakrishnan and Leung [2].

For the extended type I generalized logistic distribution given in (2.4), we obtained the characteristic function as

$$
\phi_{X}(t)=\frac{\lambda^{-i t} \Gamma(p+i t) \Gamma(1-i t)}{\Gamma(p)} .
$$

This characteristic function and the cumulative distribution function in (2.5) are important tools in proving some theorems that characterize the extended type I generalized logistic distribution as we will see in the next section.

3. Some theorems that relate the extended type I generalized logistic to some other distributions. We state some theorems and prove them in this section.

THEOREM 3.1. Let $Y$ be a continuously distributed random variable with probability density $f_{Y}(y)$. Then the random variable $X=-\ln \left[\lambda\left(e^{Y}-1\right)\right]$ has an extended type I generalized logistic distribution with parameters $p$ and $\lambda$ if and only if $Y$ has an exponential probability distribution with parameter $p$.

Proof. If $Y$ has exponential distribution with parameter $p$, then the probability density function of $Y$ is

$$
f_{Y}(y ; p)=p e^{-p y}, \quad y>0, p>0 .
$$

Then $x=-\ln \left[\lambda\left(e^{y}-1\right)\right]$ implies that $y=\ln \left(\left(e^{-x}+\lambda\right) / \lambda\right)$. Therefore

$$
f_{X}(x)=\left|\frac{d y}{d x}\right| f_{Y}(y)=\frac{p \lambda^{p} e^{-x}}{\left(\lambda+e^{-x}\right)^{p+1}}, \quad-\infty<x<\infty,
$$

which is the extended type I generalized logistic density function.

Conversely, if $X$ is an extended type I generalized logistic random variable, then $x=-\ln \left[\lambda\left(e^{y}-1\right)\right]$ implies that

$$
\begin{gathered}
\frac{d x}{d y}=\frac{-e^{y}}{\left(e^{y}-1\right)}, \\
f_{Y}(y)=\left|\frac{d x}{d y}\right| f_{X}(x)=p e^{-p y}, \quad y>0, p>0 .
\end{gathered}
$$

Since this is the probability density function of an exponential random variable $Y$ with parameter $p$, the proof is complete.

THEOREM 3.2. Suppose $Y_{1}$ and $Y_{2}$ are independently distributed random variables. If $Y_{1}$ has the gamma distribution with probability density

$$
h_{1}\left(y_{1}\right)=\frac{\lambda^{p}}{\Gamma(p)} y_{1}^{p-1} e^{-\lambda y_{1}}, \quad y_{1}>0,
$$

and $Y_{2}$ has the exponential distribution with probability density

$$
h_{2}\left(y_{2}\right)=e^{-y_{2}}, \quad y_{2}>0
$$


then the random variable $X=\ln Y_{1}-\ln Y_{2}$ has an extended type I generalized logistic distribution with parameters $p$ and $\lambda$.

Proof. Let $Y_{1}$ and $Y_{2}$ be independent random variables with probability density functions $h_{1}$ and $h_{2}$, respectively. The characteristic function of $\ln Y_{1}$ is given by

$$
\phi_{\ln Y_{1}}(t)=\int_{0}^{\infty} e^{i t \ln Y_{1}} \frac{\lambda^{p}}{\Gamma(p)} y_{1}^{p-1} e^{-\lambda y_{1}} d y_{1}=\frac{\lambda^{-i t} \Gamma(p+i t)}{\Gamma(p)} .
$$

Similarly, the characteristic function of $-\ln Y_{2}$ is given by

$$
\phi_{-\ln Y_{2}}(t)=\int_{0}^{\infty} e^{-i t \ln Y_{2}} e^{-y_{2}} d y_{2}=\Gamma(1-i t) .
$$

Since the characteristic function of the extended type I generalized logistic distribution given in (2.6) is the product of (3.6) and (3.7), the theorem follows.

THEOREM 3.3. Let $Y$ be a continuously distributed random variable with probability density function $f_{Y}(y)$. Then the random variable $X=-\ln (Y-\lambda)$ is an extended type I generalized logistic random variable if and only if $Y$ follows a generalized Pareto distribution with parameters $\lambda$ and $p$ which are positive real numbers.

Proof. If $Y$ has the generalized Pareto distribution with parameters $\lambda$ and $p$, then

$$
f_{Y}(y ; \lambda, p)=\frac{p \lambda^{p}}{y^{p+1}}, \quad y>\lambda
$$

(see McDonald and $\mathrm{Xu}[7])$. Then $x=-\ln (y-\lambda)$ implies that $y=\lambda+e^{-x}$ and the Jacobian of the transformation is $|J|=e^{-x}$. Therefore,

$$
f_{X}(x)=|J| f_{Y}(y)=\frac{p \lambda^{p} e^{-x}}{\left(\lambda+e^{-x}\right)^{p+1}}
$$

which is the extended type I generalized logistic density function.

Conversely, if $X$ is an extended type I generalized logistic random variable with probability distribution function shown in (2.5), then

$$
\begin{aligned}
F_{Y}(y) & =\operatorname{pr}[Y \leq y]=\operatorname{pr}\left[\left(\lambda+e^{-x}\right) \leq y\right] \\
& =1-F_{X}(-\ln (y-\lambda))=1-\left(\frac{\lambda}{y}\right)^{p} .
\end{aligned}
$$

Since (3.11) is the cumulative distribution function for the generalized Pareto distribution given in (3.8), the proof is complete.

THEOREM 3.4. The random variable $X$ is extended type I generalized logistic with probability distribution function $F$ given in (2.5) if and only if $F$ satisfies the homogeneous differential equation

$$
\left(\lambda+e^{-x}\right) F^{\prime}-p e^{-x} F=0,
$$

where the prime denotes differentiation, $F$ denotes $F(x)$, and $F^{\prime}$ denotes $F^{\prime}(x)$. 
Proof. Since

$$
F=\frac{\lambda^{p}}{\left(\lambda+e^{-x}\right)^{p}},
$$

if the random variable $X$ is an extended type I generalized logistic distribution, it is easily shown that $F$ above satisfies (3.12).

Conversely, we assume that $F$ satisfies (3.12). Separating the variables in (3.12) and integrating, we have

$$
\ln F=-p \ln \left(\lambda+e^{-x}\right)+\ln k
$$

where $k$ is a constant. Obviously, from (3.14),

$$
F=\frac{k}{\left(\lambda+e^{-x}\right)^{p}} .
$$

The value of $k$ that makes $F$ a distribution function is $k=\lambda^{p}$.

4. Possible application of Theorem 3.4. From (3.12), we have

$$
x=\ln \left(\frac{p F-F^{\prime}}{\lambda F^{\prime}}\right) .
$$

Thus, the importance of Theorem 3.4 lies in the linearizing transformation (4.1). The transformation (4.1) which we call "extended type I generalized logit transform" can be regarded as an extended type I generalization of Berkson's logit transform in [3] for the ordinary logistic model.

Therefore, in the analysis of bioassay and quantal response data, if model (2.4) is used, what Berkson's logit transform does for the ordinary logistic model can be done for the extended type I generalized logistic model (2.4) by the transformation (4.1).

\section{REFERENCES}

[1] N. Balakrishnan and M. Y. Leung, Means, variances and covariances of order statistics, BLUEs for the type I generalized logistic distribution, and some applications, Comm. Statist. Simulation Comput. 17 (1988), no. 1, 51-84.

[2] _ Order statistics from the type I generalized logistic distribution, Comm. Statist. Simulation Comput. 17 (1988), no. 1, 25-50.

[3] J. Berkson, Application of the logistic function to bioassay, J. Amer. Statist. Assoc. 39 (1944), 357-365.

[4] _ Why I prefer logits to probits, Biometrics 7 (1951), 327-339.

[5] _ A statistically precise and relatively simple method of estimating the bio-assay and quantal response, based on the logistic function, J. Amer. Statist. Assoc. 48 (1953), 565-599.

[6] E. O. George and M. O. Ojo, On a generalization of the logistic distribution, Ann. Inst. Statist. Math. 32 (1980), no. 2, 161-169.

[7] J. B. McDonald and Y. J. Xu, A generalization of the beta distribution with applications, J. Econometrics 66 (1995), 133-152.

[8] M. O. Ojo, A remark on the convolution of the generalized logistic random variables, to appear in ASSET series A.

[9] _ _ Analysis of some prison data, J. Appl. Stat. 16 (1989), no. 6, 377-383. 
[10] Some relationships between the generalized logistic and other distributions, Statistica (Bologna) 57 (1997), no. 4, 573-579.

[11] Approximations of the distribution of the sum of random variables from the generalized logistic distribution, Kragujevac J. Math. 24 (2002), 135-145.

[12] P. F. Verhulst, Recherches mathematiques sur la loi d'accresioement de la population, Nouveaux memoires de l'Academie Royale des Sciences et Belles-Lettres de Bruxelles 18 (1845), 1-38 (French).

[13] J.-W. Wu, W.-L. Hung, and H.-M. Lee, Some moments and limit behaviors of the generalized logistic distribution with applications, Proc. Natl. Sci. Counc. ROC(4) 24 (2000), no. 1, $7-14$.

A. K. Olapade: Department of Mathematics, Obafemi Awolowo University, Ile-Ife 220005, Nigeria

E-mail address: akolapad@oauife.edu.ng 


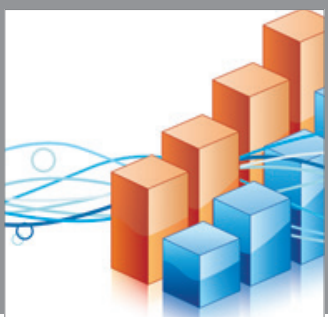

Advances in

Operations Research

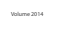

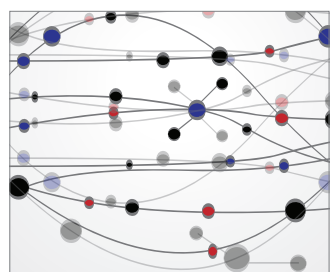

\section{The Scientific} World Journal
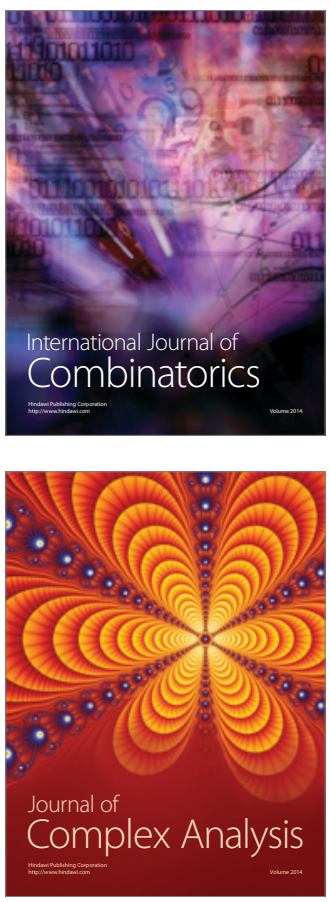

International Journal of

Mathematics and

Mathematical

Sciences
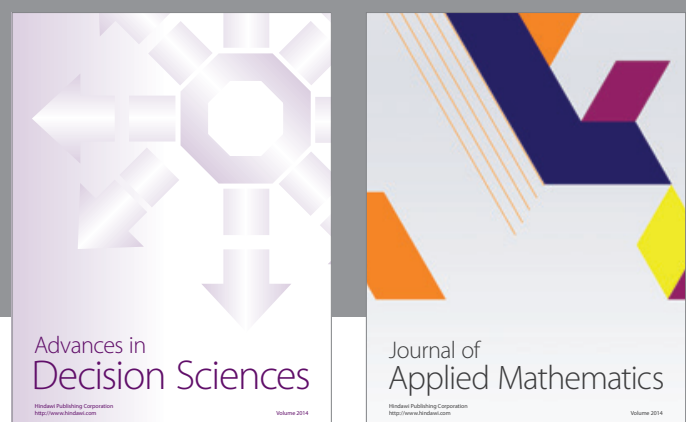

Journal of

Applied Mathematics
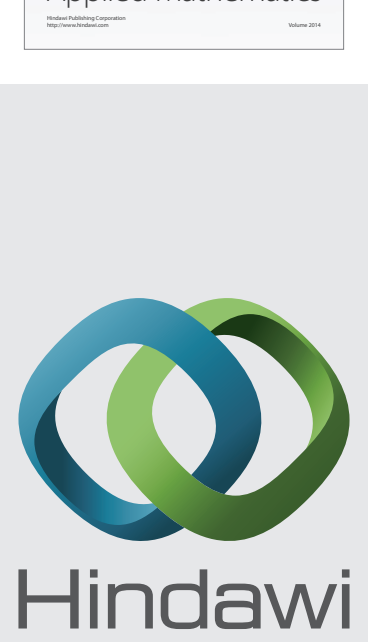

Submit your manuscripts at http://www.hindawi.com
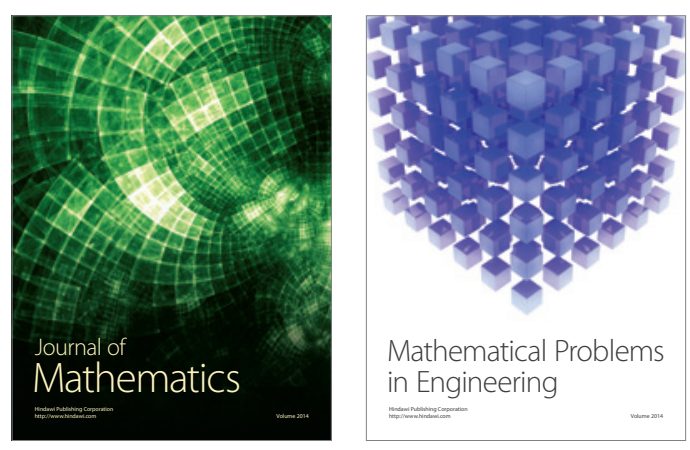

Mathematical Problems in Engineering
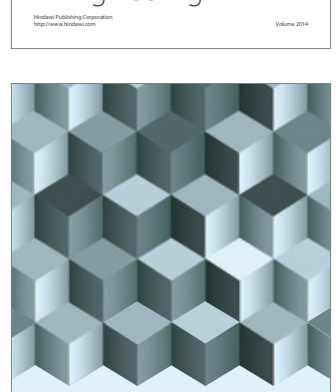

Journal of

Function Spaces
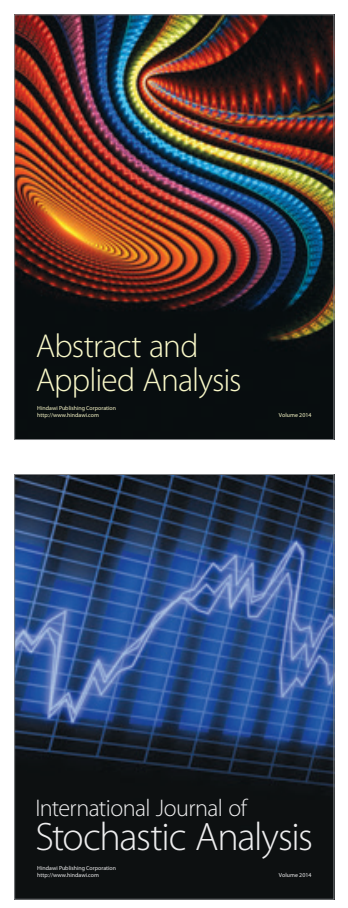

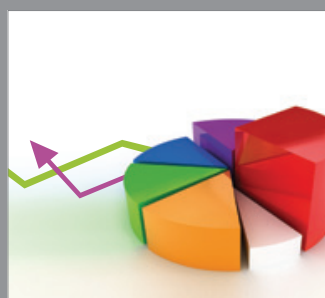

ournal of

Probability and Statistics

Promensencen
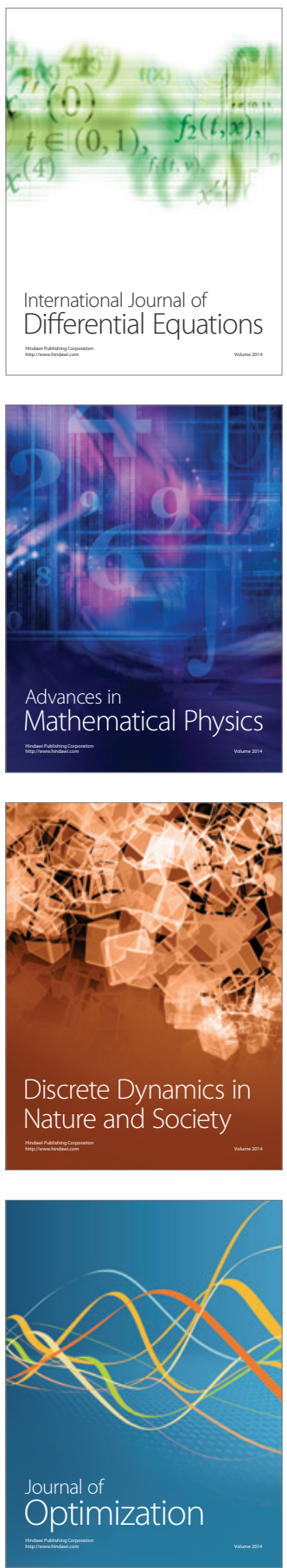